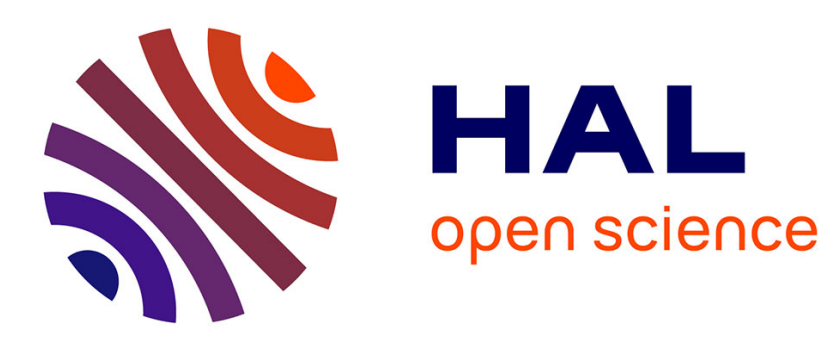

\title{
Training Effect in an Exchange Bias System: The Role of Interfacial Domain Walls
}

Thomas Hauet, J.A. Borchers, Ph Mangin, Y Henry, S Mangin

\section{To cite this version:}

Thomas Hauet, J.A. Borchers, Ph Mangin, Y Henry, S Mangin. Training Effect in an Exchange Bias System: The Role of Interfacial Domain Walls. Physical Review Letters, 2006, 10.1103/PhysRevLett.96.067207 . hal-01345166

\section{HAL Id: hal-01345166 https://hal.science/hal-01345166}

Submitted on 13 Jul 2016

HAL is a multi-disciplinary open access archive for the deposit and dissemination of scientific research documents, whether they are published or not. The documents may come from teaching and research institutions in France or abroad, or from public or private research centers.
L'archive ouverte pluridisciplinaire HAL, est destinée au dépôt et à la diffusion de documents scientifiques de niveau recherche, publiés ou non, émanant des établissements d'enseignement et de recherche français ou étrangers, des laboratoires publics ou privés. 


\title{
Training Effect in an Exchange Bias System: The Role of Interfacial Domain Walls
}

\author{
T. Hauet, ${ }^{1}$ J. A. Borchers, ${ }^{2}$ Ph. Mangin, ${ }^{3}$ Y. Henry, ${ }^{4}$ and S. Mangin ${ }^{1,5}$ \\ ${ }^{1}$ LPM, Université Henri Poincaré-Nancy I, Boîte Postale 239, F-54506 Vandoeuvre Cedex, France \\ ${ }^{2}$ NIST Center for Neutron Research Building 235, E151 100 Bureau Drive, Stop 8562 Gaithersburg, Maryland 20899-8562, USA \\ ${ }^{3}$ Laboratoire Leon Brillouin (LLB), UMR 12 CEA/CNRS, Bat. 563 CEA Saclay Gif sur Yvette, France \\ ${ }^{4}$ IPCMS-GEMM, 23 rue du Loess, Boîte Postale 43, 67034 Strasbourg Cedex 2, France \\ ${ }^{5}$ Hitachi Gst, 650 Harry Road, San Jose, California 95120, USA
}

(Received 27 September 2005; published 17 February 2006)

\begin{abstract}
Polarized neutron reflectivity (PNR) is used to obtain the magnetic depth profile of an antiferromagnetically coupled ferrimagnetic/ferrimagnetic bilayer, $\mathrm{Gd}_{40} \mathrm{Fe}_{60} / \mathrm{Tb}_{12} \mathrm{Fe}_{88}$. This system shows a transition from positive to negative exchange bias field $H_{E}$ as the cooling field $H_{\mathrm{cf}}$ is increased from small to large positive value. It also exhibits training behavior upon field cycling which affects $H_{E}$ and the coercive field $H_{C}$. From the PNR measurements at room temperature and at $15 \mathrm{~K}$, we confirm that the magnetic configuration inside the $\mathrm{TbFe}$ layer is frozen when the sample is cooled in various $H_{\mathrm{cf}}$. The thickness and pitch of the magnetic twist inside the TbFe layer depend on $H_{\mathrm{cf}}$ and give rise to the observed differences in the bias field. Irreversible reorganization of the TbFe magnetization at the interface occurs upon GdFe magnetization reversal and is found to explain the training effect as well as the overall reduction in coercivity.
\end{abstract}

DOI: 10.1103/PhysRevLett.96.067207

Exchange bias in a ferromagnetic/antiferromagnetic (FM/AF) systems was discovered in 1957 [1] and has been extensively studied during the past 10 years [2], partly because of its applications in spin-electronic devices (GMR heads, sensors, etc.). Among the most fascinating FM/AF systems are $\mathrm{FeF}_{2} / \mathrm{Fe}$ and $\mathrm{MnF}_{2} / \mathrm{Fe}$ [3]. Below the Blocking temperature $\left(T_{B}\right)$ they show a variety of unusual effects related to antiferromagnetic exchange coupling between the two materials [3]. The hysteresis loop corresponding to the magnetization reversal of the ferromagnetic layer is shifted along the field axis toward $H_{E}$ (the exchange bias field), and also along the magnetization axis leading to a "magnetization shift" $M_{\text {Shift }}$ [4]. These effects, as well as variations in the coercivity $H_{C}$, are shown to depend on the experimental procedure used to cool the system. Specifically, $H_{E}, H_{C}$, and $M_{\text {Shift }}$ depend strongly on the field $\left(H_{\mathrm{cf}}\right)$ applied as the sample is cooled through $T_{B}$ [4]. Despite great efforts and the use of a variety of experimental techniques $[3,4]$ to understand and relate all those phenomena, mechanisms explaining the behaviors of antiferromagnetically coupled biased layers are still strongly debated. Another interesting phenomenon, known as the "training effect," is observed in FM/AF systems when they are field cycled. In this case, the exchange bias field is found to vary with the number of hysteresis loops $[5,6]$. To our knowledge this effect was only observed in ferromagnetically coupled systems. It is generally believed that this effect comes from irreversible changes in the AF layer. However, no direct observation of an AF metastable state has been made to date because of the experimental difficulty in probing the magnetic configuration of the $\mathrm{AF}$ layer. $\mathrm{GdFe} / \mathrm{TbFe}$ ferrimagnetic bilayers have been shown to be an ideal model system to study magnetic configurations in exchange-biased bilayers [7]. Specifically, for alloy compositions which lead to antiferromagnetic inter-
PACS numbers: 75.25.+z, 75.60.Ch, 75.60.Lr, 75.70.Cn

layer exchange coupling, the behaviors are astonishingly similar to those measured for $\mathrm{Fe} / \mathrm{FeF}_{2}[3,8]$.

In a previous paper, magnetization measurements supported by a micromagnetic simulation indicated that the observed evolution of $H_{E}$ and $M_{\text {Shift }}$ with the cooling field $H_{\text {cf }}$ [8] depends upon the characteristics of the frozen $\mathrm{TbFe}$ spin twists. The simulation is obtained by minimizing Zeeman, anisotropy, and exchange energies for a zerotemperature one-dimensional chain of spins. In this Letter we combine both conventional magnetization measurements and polarized neutron reflectivity (PNR), which is uniquely sensitive to the magnetic depth profile, to probe directly the frozen $\mathrm{TbFe}$ spin configurations. We confirm that the spin twists have characteristics that are consistent with expectations from our previous work [8]. Moreover, we demonstrate that the training effect, which includes an overall decrease in the coercivity, originates from irreversible changes in the spin configuration within the $\mathrm{TbFe}$ pinning layer.

As background, we review the features of our Glass $/ \mathrm{Gd}_{40} \mathrm{Fe}_{60}(100 \mathrm{~nm}) / \mathrm{Tb}_{12} \mathrm{Fe}_{88}(50 \mathrm{~nm}) / \mathrm{Al}(4.5 \mathrm{~nm}) /$ $\mathrm{Al}_{2} \mathrm{O}_{3}(3.5 \mathrm{~nm})$ system. Both $\mathrm{Gd}_{40} \mathrm{Fe}_{60}$ and $\mathrm{Tb}_{12} \mathrm{Fe}_{88}$ are amorphous ferrimagnetic alloys in which the $\mathrm{Fe}$ and rareearth magnetic moments are antiferromagnetically coupled. For those compositions, the two alloys magnetization are antiferromagnetically exchange coupled. In this case, $\mathrm{TbFe}$ is a soft magnetic material at $300 \mathrm{~K}$ but becomes hard upon cooling. However, GdFe is soft at all temperatures and exhibits an in-plane macroscopic anisotropy axis [9].

Analogous to a classic exchange-spring magnet [10], the competition among the anisotropies, interlayer exchange coupling, and Zeeman energies in our $\mathrm{GdFe} / \mathrm{TbFe}$ bilayer gives rise to a rich magnetic phase diagram in which spin twists or domain walls form near the interface. 
PNR is ideally suited for imaging vertical domain walls in ferromagnetic and ferrimagnetic multilayers [11,12], as it provides a depth profile of both the sample structure and the vector magnetization. PNR measurements were performed at the NG-1 reflectometer [13] at the NIST Center for Neutron Research using a neutron wavelength of $4.75 \AA$. For these experiments, the neutron polarization direction and the applied field were maintained parallel to the GdFe uniaxial anisotropy direction in the plane of the sample. A supermirror polarizer and analyzer selected one of the spin states of the incident and scattered neutrons, respectively. The reflectivity data were corrected for the polarization efficiencies [13], which exceeded 96\%, as well as for instrumental background. We measured all four PNR reflectivities, $R^{++}, R^{--}, R^{+-}$, and $R^{-+}$, where the + and - signs designate, respectively, parallel and antiparallel polarizations of the incident and reflected neutrons relative to the field. The $R^{++}$and $R^{--}$nonspin flip reflectivities contain contributions from both the chemical film structure and the component of the magnetization parallel to the applied field. The spin flip reflectivities $R^{+-}$and $R^{-+}$depend on the average component of magnetization perpendicular to the field [13]. Depth-dependent magnetic and structural properties can be deduced by fitting PNR data with a model for the scattering length density $[13,14]$. The value of the structural as well as the magnetic parameters necessary to the fit were measured or calculated independently prior to the PNR measurements. These parameters were then optimized by fitting the wellknown antiparallel magnetic configuration at 30 Oe. The following fits then permitted us to determine only the magnetic configuration. All fits were found to minimize a $\chi^{2}$ value lower than 10 [14]. While the fits are particularly sensitive to the magnetization of the entire $\mathrm{TbFe}$ layer as well as the GdFe magnetization close to the $\mathrm{TbFe} / \mathrm{GdFe}$ interface, they are much less sensitive to $\mathrm{GdFe}$ moments closest to the substrate due to the large $\mathrm{Gd}$ absorption. Moreover, a strong decrease of the last $\mathrm{TbFe}$ moment at the interface is observed at low temperature. This effect is certainly related to the magnetic roughness of our amorphous bilayer.

Bulk magnetization measured and calculated are plotted in Fig. 1(a) as a function of the field for the $\mathrm{GdFe} / \mathrm{TbFe}$ sample at $300 \mathrm{~K}$. The corresponding magnetic depth profile obtained from PNR fits and the micromagnetic calculation are plotted on Fig. 1(b). The good agreement between measurements and calculation confirms the presence of the magnetic states identified previously [8]. The field dependence of the magnetic configuration in the bilayer can thus be summarized as follows. In fields smaller than $150 \mathrm{Oe}$, the $\mathrm{TbFe}$ magnetization is reversed relative to the applied field. This type of reversal gives rise to two symmetric magnetization drops in the hysteresis loop shown in Fig. 1(a). For fields larger than 150 Oe, an interface domain wall (IDW) forms due to the competition between the

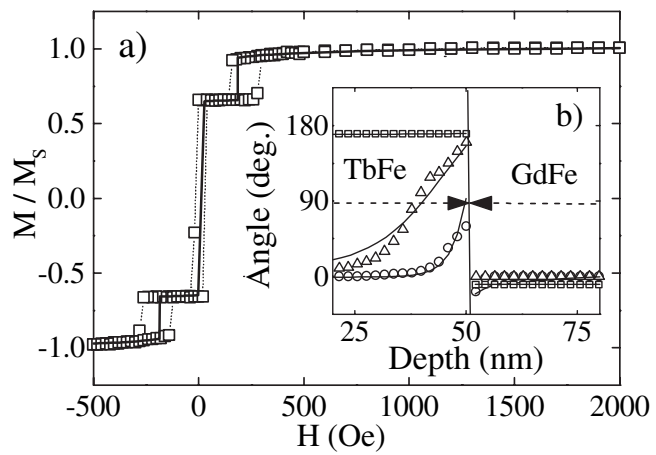

FIG. 1. (a) Normalized magnetization $\left(M / M_{S}\right)$ versus field $(H)$ applied along the easy axis for the $\mathrm{GdFe} / \mathrm{TbFe}$ bilayer at $300 \mathrm{~K}$. The line corresponds to the result of the micromagnetic simulation. (b) Angle between the magnetization and the positive field direction as a function of the depth for different fields. The top TbFe surface corresponds to the origin, and the interface (vertical line) is located at a $50 \mathrm{~nm}$ depth. The circle, triangle, and square symbols correspond, respectively, to the configurations for fields of $7 \mathrm{kOe}, 200 \mathrm{Oe}$, and $30 \mathrm{Oe}$. The lines correspond to the result of the micromagnetic simulation.

$\mathrm{GdFe} / \mathrm{TbFe}$ antiferromagnetic coupling and the Zeeman energy. In general, the IDW allows for (nearly) antiparallel alignment of the $\mathrm{GdFe}$ and $\mathrm{TbFe}$ moments near the interface and for parallel alignment of the remaining GdFe and $\mathrm{TbFe}$ moments relative to the applied field. The IDW has two characteristics: (1) It is mainly located in the TbFe layer due to its lower net magnetization. (2) Its thickness decreases as the applied field increases, an effect known as DW compression [10]. During compression, the angle between the TbFe interfacial moment and the positive field direction decreases from $180^{\circ}$ to $0^{\circ}$.

As a next step we cooled the sample to $15 \mathrm{~K}$ in different fields in order to freeze the $\mathrm{TbFe}$ magnetic configurations shown in Fig. 1(b) and to probe the influence of these frozen IDWs on the reversal of the GdFe magnetization. The field was cycled twice between $+200 \mathrm{Oe}$ and -200 Oe in order to flip only the soft GdFe, and not the $\mathrm{TbFe}$. (Note that the frozen TbFe layer thus acts as a pinning layer analogous to the frozen $\mathrm{AF}$ layer in an $\mathrm{AF} /$ FM exchange-biased system.) Fig. 2(a) shows the resultant hysteresis loops for $H_{\mathrm{cf}}=200 \mathrm{Oe}$ and $7 \mathrm{kOe}$. The first and second loops can be superimposed for $H_{\mathrm{cf}}=+200 \mathrm{Oe}$, but differ dramatically for $H_{\mathrm{cf}}=7 \mathrm{kOe}$. Figure 2(b) shows $H_{E}$ and $H_{C}$ as a function of the cooling field $H_{\text {cf }}$ for the first and the second hysteresis loops. This is, to our knowledge, the first time that training effects have been observed for an antiferromagnetically coupled bilayer. In general, training may lead to a decrease of the exchange bias field [5] but also may change the sign of the exchange bias field. In our case [Fig. 2(b)], $H_{E}$ increases with $H_{\mathrm{cf}}$, with $H_{E}$ crossing zero at $H_{\mathrm{cf}}=2000 \mathrm{Oe}$. When $H_{E}$ is positive, it increases even more after training. In contrast, the coercivity is found to increase and then decrease as $H_{\mathrm{cf}}$ is increased, with a 

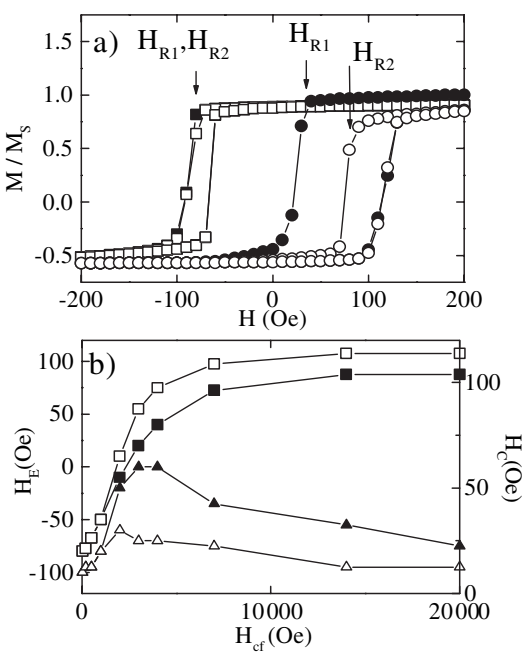

FIG. 2. (a) Normalized magnetization $\left(M / M_{S}\right)$ as a function of the field applied along the easy axis for the $\mathrm{GdFe} / \mathrm{TbFe}$ bilayer at $15 \mathrm{~K}$ after cooling from $300 \mathrm{~K}$ in $H_{\mathrm{cf}}=200 \mathrm{Oe}$ (squares) and $H_{\text {cf }}=7 \mathrm{kOe}$ (circles). Two successive hysteresis loops are shown. The first one has full symbols and the second has open symbols. $H_{R 1}$ and $H_{R 2}$ correspond, respectively, to the reversal fields for the first and the second loops. (b) Evolution of $H_{E}=$ $\left(H_{R 2}-H_{R 1}\right) / 2$ (squares) and $H_{C}=\left(H_{R 2}+H_{R 1}\right) / 2$ (up triangles) as a function of the cooling field for the first (full symbols) and second (open symbols) magnetic loop at $15 \mathrm{~K}$.

maximum coercivity coincident with $H_{E}=0 \mathrm{Oe}$. As observed for other systems $H_{C}$ is reduced after training [4].

PNR measurements were performed at $15 \mathrm{~K}$ after cooling from $300 \mathrm{~K}$ in various fields in order to link directly the evolution of $H_{E}$ and $H_{C}$ to features of the $\mathrm{TbFe}$ magnetic configuration. Fits to the low-temperature PNR data measured in $H=H_{\mathrm{cf}}$ indicate that the magnetic structure of the $\mathrm{TbFe}$ layer nearly matches the one measured at $300 \mathrm{~K}$ in $H=H_{\text {cf }}$. (We note, however, that the $\mathrm{GdFe}$ and $\mathrm{TbFe}$ moments do increase upon cooling, consistent with bulk.) Clearly, the TbFe magnetic configuration freezes when the sample is cooled from 300 to $15 \mathrm{~K}$ as expected [8].

As an example, PNR data for $H_{\mathrm{cf}}=200$ Oe are shown in Fig. 3. Similar to the +200 Oe configuration at room temperature [Fig. 1], an IDW that is approximately $35 \mathrm{~nm}$ thick lies inside the $\mathrm{TbFe}$ in fields of $+200 \mathrm{Oe}$ and $-200 \mathrm{Oe}$, and the TbFe interface moment is nearly antiparallel to the applied field [Fig. 3(b)]. This frozen TbFe configuration favors alignment of the GdFe moment parallel to the positive field direction, and a domain wall is thus forced into the GdFe layer when the field is reversed to -200 Oe [Fig. 3(b)]. The paper by Henry et al. [15] demonstrates that the field corresponding to the first reversal of the GdFe layer $\left(H_{R 1}=H_{E 1}-H_{C 1}\right)$ is determined by the orientation of the frozen $\mathrm{TbFe}$ magnetization at the $\mathrm{GdFe} / \mathrm{TbFe}$ interface. The measured reversal field, $H_{R 1}=$ $-90 \mathrm{Oe}$, is negative and has a maximum in its magnitude that is consistent with expectations for a $\mathrm{TbFe}$ interface

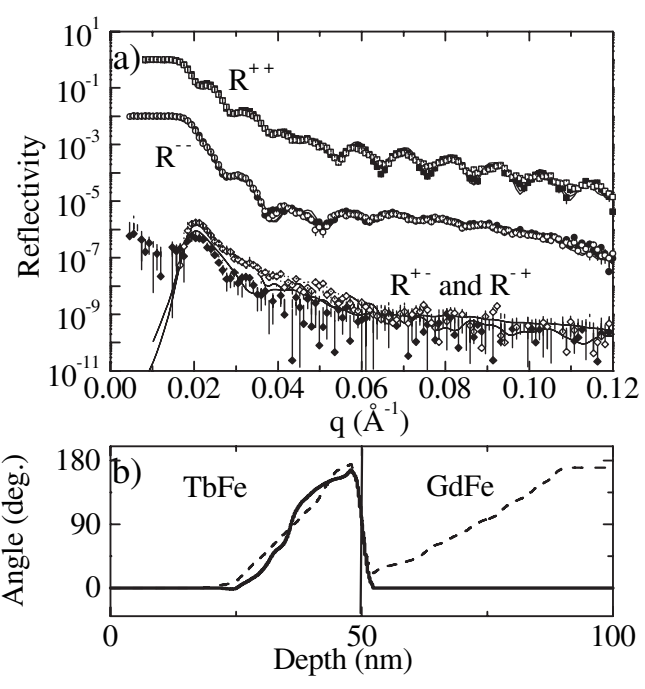

FIG. 3. (a) PNR spectra obtained after cooling the $\mathrm{GdFe} / \mathrm{TbFe}$ bilayer to $15 \mathrm{~K}$ in $H_{\mathrm{cf}}=200 \mathrm{Oe}$, for $H=+200$ Oe before any GdFe reversal (full symbols) and $H=-200$ Oe after the first $\mathrm{GdFe}$ reversal (open symbols). (b) Magnetic profile deduced from the fits in (a) at $H=+200 \mathrm{Oe}$ (solid line) and $H=$ -200 Oe (dashed line).

angle of about $180^{\circ}$. We note that no training is observed for this cooling field [Fig. 2].

In contrast, Figs. 4(a) and 4(b) show PNR spectra after field cooling in $7 \mathrm{kOe}$ to $15 \mathrm{~K}$. The training effect is obvious from the differences between the two +200 Oe data sets that were measured before and after the first hysteresis loop. Fits to the first PNR data indicate that a small IDW, with approximate thickness of $15 \mathrm{~nm}$, is
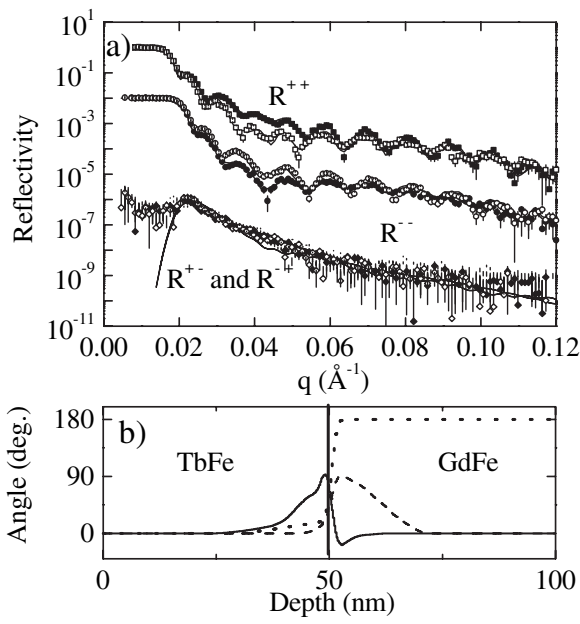

FIG. 4. (a) PNR spectra for the GdFe/TbFe bilayer obtained after cooling in $7 \mathrm{kOe}$ to $15 \mathrm{~K}$ in $H=+200$ Oe before GdFe reversal (full symbols) and $H=+200 \mathrm{Oe}$ after the first $\mathrm{GdFe}$ reversal (open symbols). (b) Magnetic profile deduced from PNR fits at $H=+200$ Oe before reversal (solid line), $H=$ -200 Oe (dotted line), and $H=+200$ Oe (dashed line) after reversal. 
present in the TbFe layer [Fig. 4(b)]. The TbFe interface moment is not parallel to its anisotropy axis, but is oriented at an angle of $70^{\circ}$ relative to the positive field direction. The measured value of $H_{R 1}=20 \mathrm{Oe}$ is positive and matches expectations [15]. Upon reversing the GdFe magnetization in a field of $-200 \mathrm{Oe}$, however, the TbFe interface moment realigns parallel to its anisotropy axis [Fig. 4(b)], such that the GdFe and TbFe layer moments are nearly antiparallel. When the field is swept back to $+200 \mathrm{Oe}$, the bottom of the GdFe magnetization is forced parallel to the positive field, while the TbFe magnetization remains uniformly pointed in the positive field direction. As a result, a domain wall forms in the GdFe layer in order to accommodate the antiferromagnetic interface coupling. The value of the reversal field of the second hysteresis loop $H_{R 2}(75 \mathrm{Oe})$ is in accord with the new orientation of the $\mathrm{TbFe}$ magnetization $\left(0^{\circ}\right)$ at the interface [15]. Overall, our PNR investigation of the training effect reveals that the $\mathrm{TbFe}$ spins become trapped in a metastable state when the sample is field cooled from 300 to $15 \mathrm{~K}$ because the anisotropy in the TbFe increases rapidly upon cooling. The frozen IDW in the TbFe layer has an energy cost that is inversely related to its thickness. For $H_{\text {cf }}=200 \mathrm{Oe}$ the $\mathrm{TbFe}$ magnetization at the $\mathrm{GdFe} / \mathrm{TbFe}$ interface is frozen parallel to its anisotropy direction.

The GdFe magnetization reversal is unfavorable and occurs at a maximum negative value of $H_{R 1}$. The reversal is thus accommodated by the formation of a domain wall in the GdFe layer which does not perturb the system sufficiently to alter the TbFe magnetic configuration. For $H_{\mathrm{cf}}=$ 7 kOe the energy cost of the TbFe IDW is larger because the $\mathrm{TbFe}$ interfacial moment is canted relative to its anisotropy axis. The GdFe magnetization reversal occurs in a positive field, and it forces the $\mathrm{TbFe}$ magnetization to align parallel to its anisotropy direction in order to minimize the energy. This new spin configuration in the TbFe layer remains unchanged with additional field cycling. The training effect is thus dominated by IDW annihilation which is more likely to take place when the IDW energy cost is large. This same mechanism gives rise to the overall reduction of the coercivity observed since GdFe reversals are accommodated by the formation of a lower-energy domain wall in the soft GdFe layer after training. While the unusual variation of the coercivity with the cooling field is not completely understood, it may be related to the $H_{\mathrm{cf}}$ dependence of the absolute difference between the angle of the $\mathrm{TbFe}$ interfacial moment and TbFe's uniaxial anisotropy direction.
In conclusion, using PNR measurements we have determined directly the magnetic depth profile of an antiferromagnetically coupled $\mathrm{GdFe} / \mathrm{TbFe}$ bilayer, and correlated it with the observed exchange-biasing. We confirmed that an interface domain wall, mainly located in the TbFe layer, freezes upon field cooling. The IDW characteristics are consistent with expectations from theory and other measurement techniques [15]. More important, PNR measurements reveal that the training effect in this system originates directly from irreversible changes (i.e., "annealing") of the nominally frozen IDW near the TbFe interface. After training, the free layer is consequently forced to accommodate a domain wall during field reversal. These measurements thus constitute the first direct observations of the frozen spin configuration in the pinning layer and of the dramatic changes in the magnetic structure of an exchange-biased bilayer as it undergoes training. These results have clear implications for the energetic stability of the AF spin state in typical AF/FM exchange-biased systems.

We thank D. Ligiardi for help with the experiments and F. Montaigne, E. Gauthier, and F. Greullet for help with simulations. We acknowledge C. F. Majkrzak for insightful discussions.

[1] W.H. Meiklejohn and C.P. Bean, Phys. Rev. 105, 904 (1957).

[2] A.E. Berkowitz and K. Takano, J. Magn. Magn. Mater. 200, 552 (1999); J. Nogues and I. K. Schuller, J. Magn. Magn. Mater. 192, 203 (1999); M. Kiwi, J. Magn. Magn. Mater. 234, 584 (2001).

[3] J. Nogues et al., Phys. Rev. Lett. 76, 4624 (1996); C. Leighton et al., Phys. Rev. B 65, 064403 (2002).

[4] J. Nogues et al., Phys. Rev. B 61, 1315 (2000).

[5] J. Keller et al., Phys. Rev. B 66, 014431 (2002).

[6] M. Ali et al., Phys. Rev. B 67, 172405 (2003).

[7] S. Mangin et al., Phys. Rev. Lett. 82, 4336 (1999).

[8] S. Mangin et al., Phys. Rev. B 68, 140404(R) (2003).

[9] S. Mangin et al., J. Magn. Magn. Mater. 165, 161 (1997).

[10] E. F. Kneller and R. Hawig, IEEE Trans. Magn. 27, 3588 (1991); E. E. Fullerton et al., Phys. Rev. B 58, 12193 (1998).

[11] M. R. Fitzsimmons et al., J. Magn. Magn. Mater. 271, 103 (2004).

[12] K. V. O’Donovan et al., Phys. Rev. Lett. 88, 067201 (2002).

[13] C. F. Majkrzak, Physica (Amsterdam) 221B, 342 (1996).

[14] http://www.ncnr.nist.gov/programs/reflect/

[15] Y. Henry, S. Mangin, T. Hauet, and F. Montaigne (to be published). 